transcription PCR. Gain-and loss-of-function experiments were conducted to investigate the biological functions of LINC00460 both in vitro and in vivo. The mechanism of LINC00460 action was explored through bioinformatics, RNA fluorescence in situ hybridization, chromatin immunoprecipitation assay and RNA immunoprecipitation assay.

Results LINC00460 was excessively expressed in GC tissues and correlated with advanced stage, larger tumor size and poorer prognosis in GC patients. Forced LINC00460 expression promotes proliferation, whereas loss of LINC00460 function decreased cell proliferation by blocking of cell cycle in GC cells. Mechanistically, LINC00460 could simultaneously interact with $\mathrm{EZH} 2$, a core component of polycomb repressive complex 2, and mediates the trimethylation of $\mathrm{H} 3 \mathrm{~K} 27$ at the promoter region of $\mathrm{p} 21$. In addition, rescue experiments determined that LINC00460 oncogenic function is partly dependent on repressing P21.

Conclusions Together, our results suggest that LINC00460, as a regulator of proliferation, may serve as a candidate prognostic biomarker and target for the management of GC.

\section{IDDF2019-ABS-0137 IS MICROSCOPIC COLITIS(MC) RESTRICTED TO COLON? STUDY OF ILEAL CHANGES IN PATIENTS OF MC}

Anupam Kumar Singh*, Siddharth Shukla, Rakesh Kochhar, Balaji L Bellam, Satyavathi Rana, Kaushal K Prasad, Narender Dhaka, Sarthak Malik, Neha Berry, Megha Sharma, Saroj Kant Sinha. Post Graduate Institute of Medical Education and Research, Chandigarh, India

\subsection{6/gutjnl-2019-IDDFabstracts.31}

Background Chronic diarrhea due to Microscopic colitis (MC) is often overlooked. Restriction of disease to the colon is still unclear, and on drawing analogies from Idiopathic Ulcerative Colitis any proximal disease extension may have bearing on treatment regimes. We evaluated terminal ileum using narrow band imaging (NBI), high definition white light endoscopy (HDWLE) and histopathological findings on biopsy

Methods $\underline{53}$ adults with suspected MC were enrolled. All underwent colonoscopy with ileal intubation. Segmental colonic and terminal ileum biopsies were obtained using HDWLE and NBI. Same were analysed by expert gastrointestinal histopathologist

Results In 43 patients of confirmed MC, HDWLE of ileum was normal. On NBI, intravillous capillary network was regular unbranched with semi-circular pattern in $41(95.4 \%)$ of patients of $\mathrm{MC}$ and in all controls. It was sparse and irregular in $2(4.7 \%)$ cases. Peyer's patch domes were indistinct in 5(9.4\%), normal in $38(88.4 \%)$ cases and normal in all controls $(\mathrm{p}=0.570)$. Peyer's patch vessels were regular and unbranched in $38(88.4 \%)$ cases and all controls. They were sparse and irregular in 5 cases $(11.6 \%)(p=0.570)$. Ileal histopathology revealed normal villi in 39 (90.6\%) cases, 4 had partial villous atrophy. The crypt villous ratio was grouped as $1: 1.5,1: 3,1: 4$ and $1: 5$ in 2 (4.6), 26 (60.4),11 $(25.5 \%)$ and 4 (9.3) respectively. Lymphoplasmacytic infiltrate was grouped as mild, moderate and severe, and observed in $10(23.2 \%), 2(4.6 \%)$ and 1 (2.3\%) patient respectively

Conclusions Ileal NBI findings in MC are hitherto unreported, with up to $12 \%$ showing abnormalities. Histologically up to $23 \%$ showed abnormalities in ileal biopsies. Ileal pathology may contribute to certain symptoms in MC

\section{IDDF2019-ABS-0153 CLINICAL SIGNIFICANCE OF SEPRASE AND CONNEXIN-43 EXPRESSION IN GASTRIC CARCINOMA}

${ }^{1}$ Fangrui Yin*, ${ }^{2}$ Yunqi Hua, ${ }^{2}$ Xianfeng Liu, ${ }^{2}$ Yongyan Yang, ${ }^{2}$ Chenlin Wang, ${ }^{2} \mathrm{Xi}$ Li,

${ }^{2}$ Ying Huang. 'Department of Central Laboratory, The First Affiliated Hospital of Baotou Medical College, China; ${ }^{2}$ Cancer Biotherapy Center and Oncology Department, Baotou Tumor Hospital, Clinical Oncology of Baotou Medical College, China

\subsection{6/gutjnl-2019-IDDFabstracts.32}

Background To investigate the expression of connexin43( $\mathrm{Cx} 43)$ and Seprase protein in gastric carcinoma and their clinical significance.

Methods Immunohistochemical method was used to detect the expression of Seprase and connexin 43 proteins in 45 specimens of gastric carcinoma and 15 specimens of paired adjacent normal gastric tissue. The relationship between their expression and the clinicopathologic characteristics were statistically analyzed.

Results 1: The positive rate of Seprase protein in gastric carcinoma was $71.11 \%(32 / 45)$ higher than that in normal tissue $20.00 \%(3 / 15)$ and with statistical significance $\left(\chi^{2}=6.67\right.$, $\mathrm{p}=0.01$ ); The positive rate of connexin 43 protein in gastric carcinoma was $68.89 \%(30 / 45)$ lower than that in normal tissue $100 \%(15 / 15)$ and with statistical significance $\left(\chi^{2}=12.09\right.$, $\mathrm{p}=0.001)$. 2: Connexin 43 protein was related to the age and the degree of tissue differentiation of gastric carcinoma $(\mathrm{P}<0.05)$, Seprase protein was related to the age and the subtype of gastric carcinoma patients $(\mathrm{P}<0.05) .3$ : Overall survival rates of patients with seprase and Connexin 43 expression were detected, Connexin 43 expression was an independent prognosis factor; meanwhile, seprase was correlated with the survival of the patients with gastric carcinoma in our study.

Conclusions In gastric carcinoma, Connexin 43 appeared down-expression and seprase appeared up-regulation, That may play important roles in the invasion and metastasis of gastric carcinoma, then provide a new direction for target therapy.

\section{IDDF2019-ABS-0179 THE ASSOCIATION BETWEEN COMPONENTS OF METABOLIC SYNDROME AND COLORECTAL CANCER RISK IN CHINESE MALES}

${ }^{1}$ Xin Li*, ${ }^{1}$ Hongda Chen, ${ }^{2}$ Wang Gang, ${ }^{1}$ Xiaoshuang Feng, 'Zhangyan Lyu, 'Luopei Wei, ${ }^{1}$ Yan Wen, ${ }^{3}$ Shuohua Chen, ${ }^{3}$ Shouling Wu, ${ }^{1} \mathrm{Min}$ Dai, ${ }^{1} \mathrm{Ni}$ Li, ${ }^{4} \mathrm{Jie}$ He. ${ }^{1}$ Office of Cancer Screening, National Cancer Center/National Clinical Research Center for Cancer/Cancer Hospital, Chinese Academy of Medical Sciences and Peking Union Medical College, China; ${ }^{2}$ Department of Oncology, Kailuan General Hospital, Tangshan, China; ${ }^{3}$ Health Department of Kailuan (Group), Tangshan, China; ${ }^{4}$ Department of Thoracic Surgery, National Cancer Center/National Clinical Research Center for Cancer/Cancer Hospital, Chinese Academy of Medical Sciences and Peking Union Medical College, China

\subsection{6/gutjnl-2019-IDDFabstracts.33}

Background Several researches have reported the metabolic syndrome (MetS) was related to a higher risk of colorectal cancer (CRC). However, it remains unclear to what extent the MetS components individually account for the association between MetS and CRC. To investigate the association of MetS components with the risk of CRC in Chinese males, the study was performed in the Kailuan male cohort, a large prospective cohort study. 
Methods A total of 104,333 eligible males enrolled in every 2-year health checkup were involved in the Kailuan male cohort study (2006-2015). Cox proportional hazards regression models were used to estimate the association between components of MetS and the CRC risk

Results During a median follow-up of 8.9 years, 394 CRC cases were verified over a total of $824,211.96$ person-years. Compared with males without MetS components, the HRs (95\% CI) of developing CRC for males with 1,2 and $\geq 3$ MetS components were 1.53 (1.01-2.32), 1.42 (0.94-2.14) and $1.70(1.12-2.56)$, respectively, which showed a statistically trend $(\mathrm{P}$ trend $=0.04)$ for increasing risk with an increasing number of MetS components. In addition, among the 5 single MetS components, only the waist circumference $\geq 90 \mathrm{~cm}$ showed significant risk for CRC (HR: 1.32, 95\% CI: 1.071.64). Further combination of higher waist circumference and elevated fasting blood glucose $(\geq 5.6 \mathrm{mmol} / \mathrm{L}$ or drug treatment of elevated glucose) with normal levels of the other three components showed an increased risk for CRC by $126 \%$ (95\% CI: 1.10-4.64). However, the other combinations showed non statistical significant associations.

Conclusions Our study suggests CRC risk is correlated with the number of abnormal MetS components in males. Among MetS components, higher waist circumference, particularly concurrent with elevated fasting blood glucose may be more strongly related to CRC risk than other MetS components.
IDDF2019-ABS-0182 A CLOSTRIDIA-RICH ENTEROTYPE CONTRIBUTES TO INCREASED EXCRETION OF BILE ACIDS IN DIARRHEAPREDOMINANT IRRITABLE BOWEL SYNDROME

Ling Zhao*. School of Chinese Medicine, Hong Kong Baptist University, Hong Kong

\subsection{6/gutjnl-2019-IDDFabstracts.34}

Background An excess of fecal bile acids (BAs) is thought to be one of the peripheral mechanisms for diarrhea-predominant irritable bowel syndrome (IBS-D). However, the factors causing excessive BA excretion remains unclear. Given the importance of gut microbiota in BA metabolism, we hypothesized that gut dysbiosis might contribute to excessive BA excretion in IBS-D.

Methods Metabolomic and metagenomic analyses were performed of specimens from 290 IBS-D patients and 89 healthy volunteers. By transplanting human microbiota and manipulating specific bacterial species in mice, the effects of microbiota on host BA metabolism were assessed at metabolic, genetic and protein levels. Effects of individual and mixed BAs on enterohepatic feedback pathways were also tested in vitro and in vivo.

Results Total fecal BAs were excessively excreted in 71 of 290 IBS-D patients (grouped as $\mathrm{BA}^{+} \mathrm{IBS}-\mathrm{D}$ ) who also have excessive $7 \alpha$-hydroxy-4-cholesten-3-one (C4) but deficient fibroblast
A

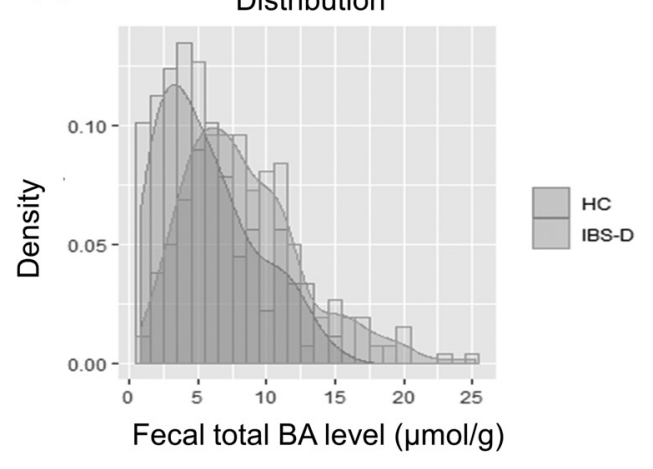

D

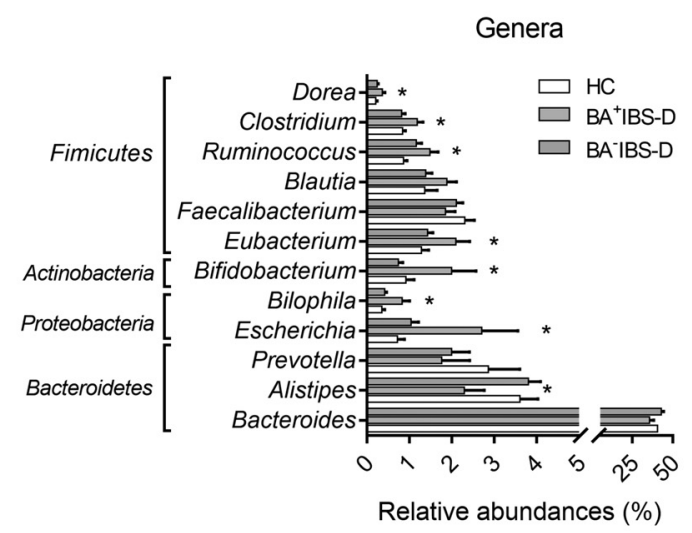

B

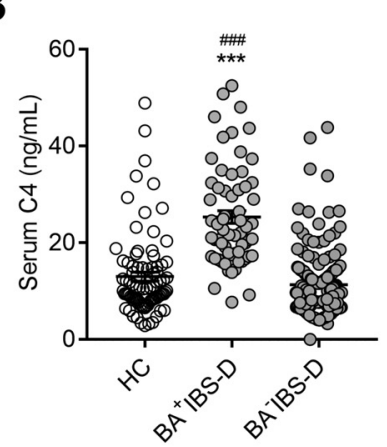

E

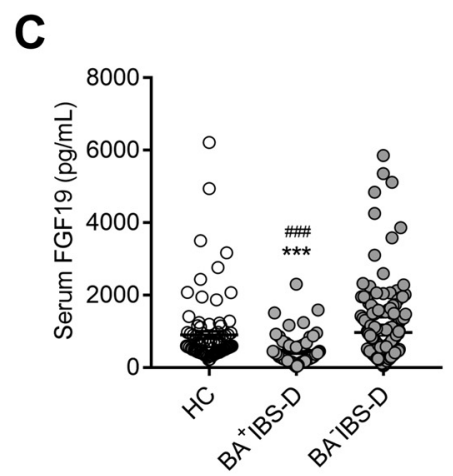

Correlation coefficient

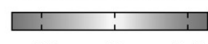

$\begin{array}{lll}-0.2 & 0 & 0.2\end{array}$

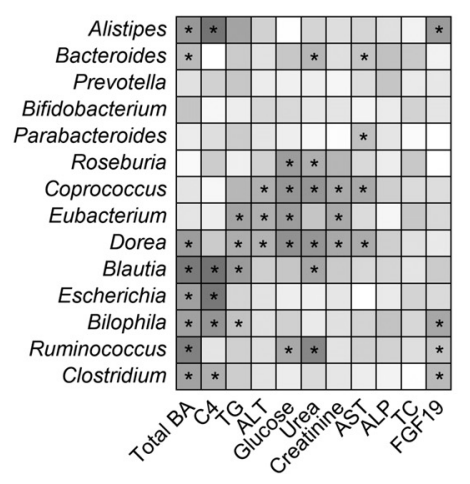

Abstract IDDF2019-ABS-0182 Figure 1 